\title{
The transcription factor RFX5 is a transcriptional activator of the TPP1 gene in hepatocellular carcinoma
}

\author{
YANGJING ZHAO ${ }^{1,2^{*}}$, XINGWANG XIE $^{2 *}$, WEIJIA LIAO ${ }^{3}$, HENGHUI ZHANG $^{2}, \mathrm{HUI} \mathrm{CAO}^{4}$, \\ RAN FEI $^{2}$, XUEYAN WANG $^{2}$, LAI WEI $^{2}$, QIXIANG SHAO ${ }^{1}$ and HONGSONG CHEN ${ }^{2}$ \\ ${ }^{1}$ Department of Immunology, and The Key Laboratory of Laboratory Medicine of Jiangsu Province, \\ School of Medicine, Jiangsu University, Zhenjiang, Jiangsu 212013; ${ }^{2}$ Peking University People's Hospital, \\ Peking University Hepatology Institute, Beijing Key Laboratory of Hepatitis C and \\ Immunotherapy for Liver Disease, Beijing 100044; ${ }^{3}$ Laboratory of Hepatobiliary and Pancreatic Surgery, \\ The Affiliated Hospital of Guilin Medical University, Guilin, Guangxi 541001; ${ }^{4}$ Department of Pathology, \\ The First Affiliated Hospital of Zhejiang University, Hangzhou, Zhejiang 310003, P.R. China
}

Received June 7, 2016; Accepted October 31, 2016

DOI: $10.3892 / o r .2016 .5240$

\begin{abstract}
Regulatory factor X-5 (RFX5) was previously characterized as an essential and highly specific regulator of major histocompatibility class II (MHCII) gene expression in the immune system. We found that RFX5 is significantly upregulated in hepatocellular carcinoma (HCC) tumors and cell lines compared with non-tumor tissues in mRNA expression levels, but it fails to induce the expression of MHCII. However, RFX5 can strongly bind to the tripeptidyl peptidase 1 (TPP1)
\end{abstract}

Correspondence to: Professor Qixiang Shao, Department of Immunology, and The Key Laboratory of Laboratory Medicine of Jiangsu Province, School of Medicine, Jiangsu University, 301 Xuefu Road, Zhenjiang, Jiangsu 212013, P.R. China

E-mail: shao_qx@ujs.edu.cn

Professor Hongsong Chen, Peking University People's Hospital, Peking University Hepatology Institute, Beijing Key Laboratory of Hepatitis C and Immunotherapy for Liver Disease, 11 Xizhimen South Street, Beijing 100044, P.R. China

E-mail: chenhongsong2999@163.com

${ }^{*}$ Contributed equally

Abbreviations: RFX5, regulatory factor X-5; TPP1, tripeptidyl peptidase 1; HCC, hepatocellular carcinoma; MHC, major histocompatibility class; TFs, transcription factors; DBD, DNA-binding domain; RFXANK/B, RFX containing ankyrin repeats; RFXAP, RFX-associated protein; NF-Y, nuclear factor Y; X2BP, X2 box binding proteins; CIITA, class II transactivator; SCID, severe combined immunodeficiency; ENCODE, the Encyclopedia of DNA Elements; TCGA, The Cancer Genome Atlas; ChIP, chromatin immunoprecipitation assay; OST, overall survival time; BLS, bare lymphocyte syndrome; RLA, relative luciferase activity

Key words: hepatocellular carcinoma, regulatory factor X-5, tripeptidyl peptidase 1, major histocompatibility class II genes, transcriptional activator promoter region and then increase its transcriptional activity. We also found that manipulation the expression of RFX5 can significantly affect the expression of TPP1 in HepG2, which suggested that RFX5 can transcriptionally activate TPP1 in HCC. Moreover, TPP1 is overexpressed in HCC tissues and significantly correlated with poor prognosis of HCC patients, suggesting that it may have potential biological implications in $\mathrm{HCC}$.

\section{Introduction}

Regulatory factor X-5 (RFX5) belongs to the RFX family, which are winged-helix transcription factors (TFs) and characterized by a highly conserved DNA-binding domain (DBD) and dimerization motifs $(1,2)$. RFX5 comprises the transcription factor complex RFX together with two other subunits, RFXANK/B and RFXAP. The most well recognized function of RFX5 and the RFX complex is to bind to MHCII gene cis-acting elements the S-Y boxes and to transactivate these genes by collaborating with NF-Y, X2BP and CIITA (3-8). Previous studies have found that RFX5 is not only expressed in MHCII-positive cells but also in MHCII-negative cells (3). Moreover, our previous study found that RFX5 could be overexpressed in hepatocellular carcinoma cells that lack MHCII expression. These phenomena suggest that RFX5 may regulate the expression of non-MHCII genes, for example by transactivating $\mathrm{MHCI}$ and $\beta 2$-microglobulin and repressing collagen gene $(9,10)$. However, less effort has been made to uncover the non-MHCII transactivating target genes of RFX5 and their significance.

Tripeptidyl peptidase 1 (TPP1), which encodes a lysosomal protease and can cleave substrate $\mathrm{N}$-terminal tripeptides, is known for its abolished enzyme activity that leads to lateinfantile neuronal ceroid lipofuscinosis disease (CLN2) $(11,12)$. However, little is known about its role in neoplastic diseases. In our previous study, we proposed that TPP1 was a RFX5 transactivating target gene by analyzing RFX5 ChIP-seq data from the Encyclopedia of DNA Elements (ENCODE). In this 
study, we aimed to prove that RFX5 acts as a direct positive transcriptional regulator of TPP1 in hepatocellular carcinoma (HCC).

\section{Materials and methods}

Cell culture and human tissue specimens. The HepG2 cell line was originally purchased from the American Type Culture Collection (ATCC, Manassas, VA, USA). The MHCC97H and MHCC97L cell lines were donated by the Academy of Military Medical Sciences (Beijing, China). All of the other cell lines used in this study were purchased from the Cell Bank of the Chinese Academy of Sciences (Shanghai, China). HepG2 cells were cultured with DMEM/F-12 medium (Gibco, Grand Island, NY, USA) in plastic dishes coated with collagen (Corning, Tewksbury, MA, USA). MHCC97H, MHCC97L, HuH-7 and HEK293T cells were cultured with DMEM (Gibco). SK-Hep-1 and PLC/PRF/5 cells were cultured with EMEM (Lonza, Basel, Switzerland). The media were supplemented with $10 \%$ fetal bovine serum and penicillin-streptomycin antibiotics (Gibco). The cultures were incubated in a humidified atmosphere containing $5 \% \mathrm{CO}_{2}$ at $37^{\circ} \mathrm{C}$.

Cancerous and corresponding non-cancerous liver tissues were obtained from HCC patients who underwent tumor resections at the Affiliated Hospital of Guilin Medical University (Guilin, China). All patients were histopathologically diagnosed with primary HCC. The specimens were cut into small pieces and rapidly transferred to liquid nitrogen for storage. Informed consent was obtained from all the patients. This study was approved by the Ethics Committee of Peking University People's Hospital and the Affiliated Hospital of Guilin Medical University.

RNAseq and ChIP-seq data. RNAseq data, which was measured experimentally using the Illumina HiSeq 2000 RNA Sequencing platform, from liver hepatocellular carcinoma patients (LIHC) was acquired from The Cancer Genome Atlas (TCGA) project through the UCSC Cancer Genomic Browser (https://genome-cancer.ucsc.edu/, TCGA LIHC_exp_HiSeqV2_exon, version: 2015-02-24). The exon expression value of a gene represents the mean expression value of all exons for that gene. The raw exon expression values were normalized by independently subtracting the mean of the genomic location on the fly. Anti-RFX5 ChIP-seq data from HepG2 was acquired from the ENCODE project (http:// genome.ucsc.edu/ENCODE/terms.html) (13).

Chromatin immunoprecipitation assay (ChIP). The ChIP assay was performed using the EZ-Zyme Chromatin Prep kit and EZ-ChIP Chromatin Immunoprecipitation kit (Millipore, Billerica, MA, USA) according to the manufacturer's instructions. Briefly, after crosslinking with formaldehyde (Sigma-Aldrich, St. Louis, MO, USA), HepG2 and MHCC97H cells were harvested with lysis buffer. Subsequently, chromatin was fragmented to $\sim 200$ bp pieces by enzymatic digestion. The sheared crosslinked chromatins were 'precleared' with protein $\mathrm{G}$ agarose, and $1 \%$ of the supernatant was preserved to be used as the Input control. The remaining DNA fragments were incubated overnight with rabbit anti-human RFX5 antibody (polyclonal, \#200-401-194; Rockland, Gilbertsville,
PA, USA) or normal rabbit IgG as a negative control. Each IP product was then enriched with protein $\mathrm{G}$ agarose. Finally, all of the protein/DNA complexes were reverse crosslinked to free DNA fragments and purified for ChIP-PCR analysis with primers covering TPP1 promoter sequences (ChIP P1 and ChIP P2), which are listed in Table I. PCR was performed using a $20-\mu 1$ reaction volume, and 50 cycling conditions (iCycler; Bio-Rad, Hercules, CA, USA). The PCR products were visualized with a gel documentation system (UVP, San Gabriel, CA, USA).

Plasmids. We constructed TPP1 luciferase reporter gene vectors based on the annotated status of the TPP1 core promoter region from ENCODE histone modification profiling data and the anti-RFX5 ChIP-seq data. Briefly, the active promoter region and RFX5 binding peak in the TPP1 upstream region were included in the reporter gene. Two promoter fragments were designed that extend from nucleotides -666 to $+822 \mathrm{bp}$ (TPP1 P1) and -320 to +868 bp (TPP1 P2) in the TPP1 gene (Fig. 2A). The promoter sequences were amplified from HepG2 genomic DNA using a high-fidelity DNA polymerase (Thermo Fisher Scientific, Waltham, MA, USA), designed with $\mathrm{KpnI}$ and $\mathrm{Nhe} \mathrm{I}$ restriction sites and subsequently inserted into the pGL4.10 vector (Promega, Madison, WI, USA); the results plasmids were named pGL4-TPP1 P1 and pGL4-TPP1 P2.

Two RFX5-specific shRNAs (RFK1, 5'-CCTCGGGAAC GGTCATCTAAA-3' and RFK2, 5'-CACTGCACCACAGGG TAATAA-3') and a non-silencing control (NS, 5'-CTGAGGT GATAAACAGTTACA-3') were cloned into the lentiviral shRNA expression vector HIV-H1 (GeneCopoeia, Rockville, MD, USA), which contains an H1 promoter driving the shRNA expression cassette and a GFP reporter gene.

The RFX5 ORF region was amplified from HepG2 cDNA with primers (RFX5 ORF) listed in Table I. The RFX5 ORF was fused with an $\mathrm{N}$-terminal Flag Tag, subcloned into the EcoRI/XhoI sites of the retroviral vector pMSCVneo (Takara Biomedical Technology, Beijing, China) and named RFX5-pMSCV. All of the recombinant plasmids were verified by sequencing and were prepared with the Plasmid DNA Purification kit according to the manufacturer's instructions (Macherey-Nagel, Duren, Germany).

Cell transfection and luciferase assay. For exogenous overexpression of RFX5, cells were transiently transfected with RFX5-pMSCV or pMSCV-vector using the liposome reagent Turbofect (Thermo Fisher Scientific) according to the manufacturer's instructions. Gene expression efficacy was confirmed by quantitative real-time PCR and western blotting.

For luciferase assays, cells were seeded in 24-well plates and co-transfected with $0.5 \mu \mathrm{g}$ firefly luciferase plasmid (pGL-vector, pGL4-TPP1 P1 and pGL4-TPP1 P2) and $0.5 \mu \mathrm{g}$ RFX5 expression plasmid (pMSCV-vector or RFX5-pMSCV) together with $50 \mathrm{ng}$ reference Renilla luciferase plasmid pGL4.74 (Promega). The dual luciferase activity assay was performed according to the manufacturer's instructions and detected with a liquid scintillation counter (Perkin-Elmer, Waltham, MA, USA).

shRNA gene knockdown. Lentivirus was produced by transiently co-transfecting 293T cells with shRNA plasmid and the 
Table I. Sequence of primers. ${ }^{\mathrm{a}}$

\begin{tabular}{lll}
\hline Code & \multicolumn{1}{c}{ Forward } & \multicolumn{1}{c}{ Reverse } \\
\hline ChIP-P1 & AGGATGAGGGCAAAGAGC & GAAAGGGATCACGTTGGA \\
ChIP-P2 & GTCAACTCACGTCCTCCG & CTCCCACTGAAACCCAC \\
TPP1 P1 & CGGCCGGGTACCTTCACTGCAATCTTTCG & CGGCCGGCTAGCTCAGAATTTGAGTTCGTAT \\
TPP1 P2 & CGGCCGGGTACCCCCCAGTTGGAAGTAGT & CGGCCGGCTAGCCACATCCCCTTTTAGGT \\
RFX5 ORF & CGGCCGCAATTGATGGCAGAAGATGAGCCTGATG & CGGCCGGTCGACTTATGGGGGTGTTGCTTTTGGG \\
RFX5 & GATGAGCCTGATGCTAAGAGC & CCCTCTACTTTGTTCTGCACG \\
TPP1 & GCCACCATCCAGTTACTTC & GTAGAGCCTTGGGTTGAGA \\
GAPDH & CCACATCGCTCAGACACCAT & GGCAACAATATCCACTTTACCAGAGT \\
\hline
\end{tabular}

aSequences are provided in the 5'-3' orientation. The underlined sequences are restriction endonuclease sites and protection bases.

packing helper plasmids pLP1, pLP2 and pVSV-G (Invitrogen, Carlsbad, CA, USA). Viral supernatants were harvested 48 or $72 \mathrm{~h}$ post-transfection and filtered through a $0.45-\mu \mathrm{m}$ filter (Millipore). Viral supernatants were enriched by centrifugation in a Macrosep Advance Centrifugal Device $(100 \mathrm{~K}$, Pall Corp., East Hills, NY, USA). The infectious viral titer was determined using a flow cytometry assay (BD Biosciences, flow cytometry, USA) for GFP-positive viruses in 293T cells. The viral titers were in the range of $10^{9} \mathrm{U} / \mathrm{ml}$ transducing medium. HepG2 was transduced with shRNA lentivirus at an optimal multiplicity of infection (MOI, 20:1) in the presence of $4 \mu \mathrm{g} / \mathrm{ml}$ polybrene (Sigma-Aldrich). Transfected cells were quickly selected with puromycin for 3-5 days before use. RFX5 silencing efficacy was assessed by quantitative real-time PCR.

Western blot analysis. Total protein was extracted from cells using RIPA lysis buffer and quantified using Pierce BCA Protein Assay kit according to the manufacturer's protocol (\#23225, Thermo Fisher Scientific). The protein was separated with SDS/PAGE NuPAGE Novex 4-12\% gel (Thermo Fisher Scientific) and transferred to a nitrocellulose membrane. Flag-tagged RFX5 was detected with both rabbit anti-human RFX5 antibody (polyclonal, \#200-401-194, Rockland) and mouse anti-human Flag-M2 antibody (monoclonal, \#F3165, Sigma-Aldrich). GAPDH was detected with HRP-conjugated mouse anti-human GAPDH antibody (polyclonal, \#HRP-60004, Proteintech, Chicago, IL, USA) as a loading control.

Reverse transcriptase PCR and quantitative real-time PCR. Total RNA was extracted with the TRIzol reagent (Invitrogen) and was reverse transcribed into cDNA (Applied Biosystems, Foster City, CA, USA) according to the manufacturer's instructions. The quantitative real-time PCR assay was performed with a mixture containing 50 ng cDNA template and SYBR Green I Master in a LightCycler 480 (Roche Applied Science, Mannheim, Germany) for 35 cycles. Primers for RFX5, TPP1 and GAPDH are listed in Table I. Relative gene expression was calculated with the comparative CT method and normalized against the housekeeping gene GAPDH. Each sample was tested three times.
Immunohistochemistry (IHC). We evaluated TPP1 levels by IHC in 14 paired HCC tumors and non-tumor tissues obtained from patients diagnosed at Department of Pathology of the First Affiliated Hospital of Zhejiang University (Hangzhou Zhejiang, China) in 2015. Paraffin-embedded tissue slides were heatwd with EDTA (pH 9.0) (ZSGB-BIO, Beijing, China) for antigen retrieval and incubated with rabbit anti-human TPP1 antibody (polyclonal, \#12479-1-AP, Proteintech), followed by EnVision detection system (Dako, Denmark). Sections were then counterstained with hematoxylin. The slides were observed using an Olympus AX80 digital microscopic camera system (Olympus, Tokyo, Japan). This study was approved by the Ethics Committee of Peking University People's Hospital and the First Affiliated Hospital of Zhejiang University.

Statistical analysis. Statistical analysis was performed using GraphPad Prism (GraphPad Software, Inc., USA). The unpaired t-test was used to calculate a two-tailed P-value. The Pearson correlation was used for correlation analysis. Kaplan-Meier survival analysis was used to analyze the correlation between gene expression and HCC patient prognosis. Data were considered statistically significant at $\mathrm{P}<0.05$, $\mathrm{P}<0.01, \mathrm{P}<0.001$ and $\mathrm{P}<0.0001$.

\section{Results}

RFX5 was upregulated in HCC but doed not induce MHCII expression. The RNAseq data from HCC tumor tissues and adjacent non-tumor tissues from TCGA LIHC data showed that RFX5 mRNA is significantly overexpressed in HCC compared with non-tumor tissues (Fig. 1A). The elevated RFX5 mRNA expression was further confirmed by quantitative real-time PCR analysis in an independent HCC patient cohort (Guilin cohort) (Fig. 1C, left side). Interestingly, RFX5 overexpression does not induce MHCII expression in HCC tissues and cell lines despite the classical regulator role for RFX5 in MHCII gene transcription. Only a weak correlation was observed in the expression level of RFX5 and MHCII genes, including HLA-DRA, HLA-DMA and HLA-DQB1, which was according to the RNAseq data from the TCGA LIHC data (Fig. 1B). The expression levels of RFX5 and HLA-DRA in the Guilin cohort detected by quantitative real-time PCR showed similar 
A

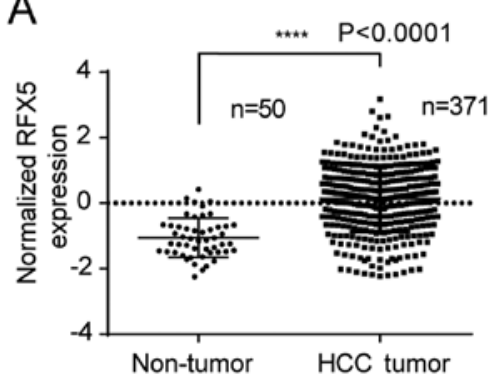

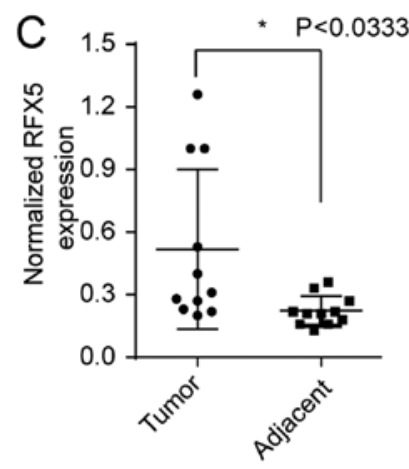
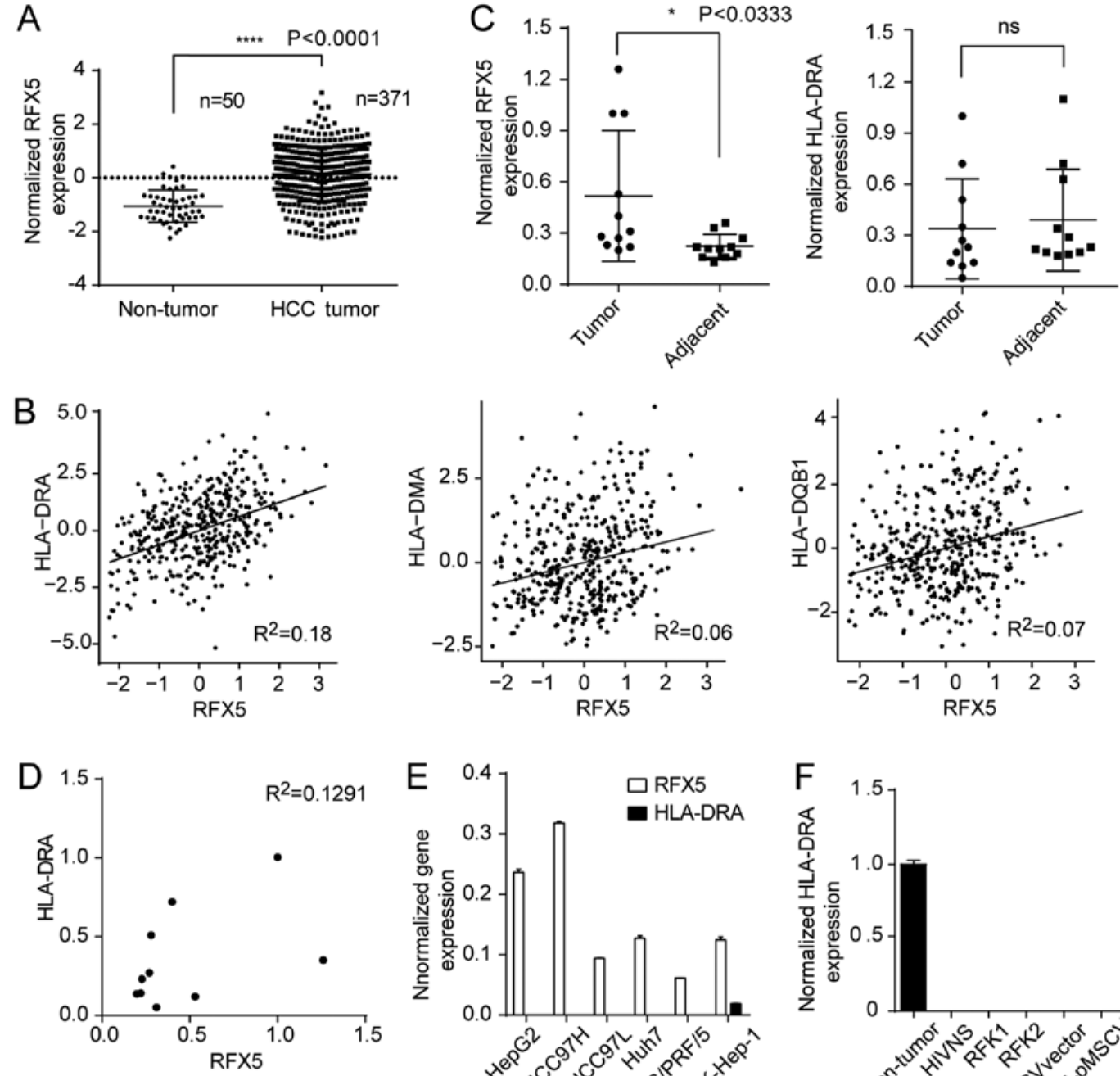
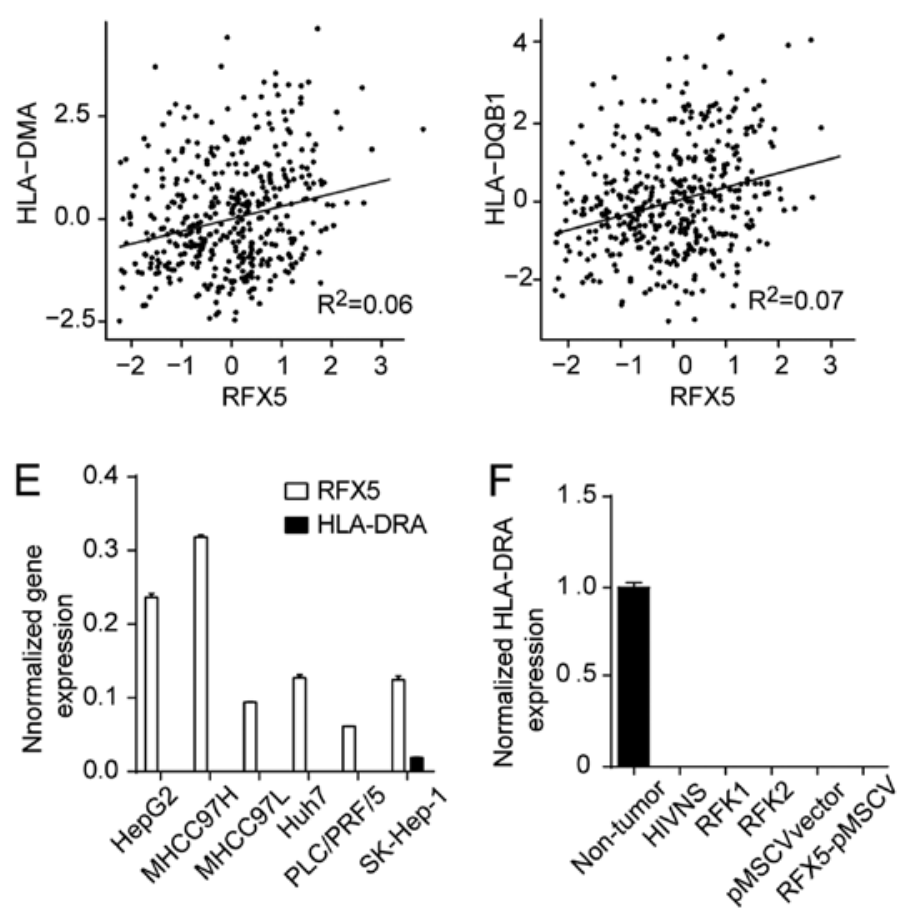

Figure 1. RFX5, but not MHCII, is overexpressed in HCC. (A) RFX5 mRNA expression levels in 421 HCC tumor and adjacent non-tumor samples from the TCGA LIHC data determined by RNAseq. (B) Correlation analysis of the mRNA expression levels for the RFX5 and MHCII genes (HLA-DRA/HLA-DMA/ HLA-DQB1) using RNAseq data from the TCGA LIHC data. (C) The mRNA expression levels of RFX5 and HLA-DRA in 11 matched cancerous and non-cancerous HCC tissues (Guilin) determined by real-time PCR. (D) Correlation analysis of the expression levels of RFX5 and HLA-DRA in HCC tissues from Guilin. (E) Real-time PCR quantification of the mRNA expression levels of RFX5 and HLA-DRA in HCC cell lines. ${ }^{*} \mathrm{P}<0.05$, ${ }^{* * * *} \mathrm{P}<0.0001$. (F) The mRNA expression of HLA-DRA in HepG2 after knockdown or overexpression of RFX5. Non-tumor, non-tumor tissue of a HCC patient; RFK1 and RFK2, two independent shRNAs targeting RFX5; HIVNS, non-silencing shRNA control; RFX5-pMSCV, RFX5 expression plasmid and pMSCV-vector, vector control.

results (Fig. 1C, right side and D). The extremely low mRNA expression of HLA-DRA in HCC cell lines by real-time PCR analysis provided more reliable evidence that high RXF5 expression levels failed to initiate the transcription of MHCII genes in HCC cells due to infiltrated immune cells, which can express both RFX5 and MHCII genes in tumor tissues, and may interfere with the results from clinical samples (Fig. 1E). Moreover, we further manipulated the expression level of RFX5 and determined its impact on the expression of MHCII genes. Strikingly, we found that neither overexpression nor silencing of RFX5 brought any change to the expression of HLA-DRA in HepG2 (Fig. 1F).

RFX5 directly binds to the TPP1 promoter. The lack of MHCII expression and the overexpression of RFX5 in HCC cells suggested that RFX5 might transcriptionally regulate other genes. Thus, we analyzed RFX5 ChIP-seq data from HepG2 cells from the ENCODE project, which contains the largest collection of high-quality ChIP-seq data sets for human TFs (14-17), to genome-widely search for other possible transcriptional target genes of RFX5. We found that RFX5 can strongly bind to the promoter region ( -668 to $+831 \mathrm{bp}$ ) of TPP1, a lysosomal enzyme tripeptidyl peptidase gene in HepG2 cells (Fig. 2A).

ChIP-PCR was performed to validate the above finding in three HCC cell lines, HepG2 and MHCC97H. Briefly, ChIP was performed with the same RFX5 antibody used in the ENCODE project, and the immunoprecipitated DNA fragments were detected by PCR with two independent primers, which cover the RFX5 binding peak in the TPP1 promoter region that was determined by the ChIP-seq in the ENCODE database (Fig. 2A). The results indicated that RFX5 could bind to the TPP1 promoter region in both HCC cell lines (Fig. 2B).

Next, we aligned the TPP1 promoter region to three of the MHCII genes and found that it contains S-X-Y motifs, which 


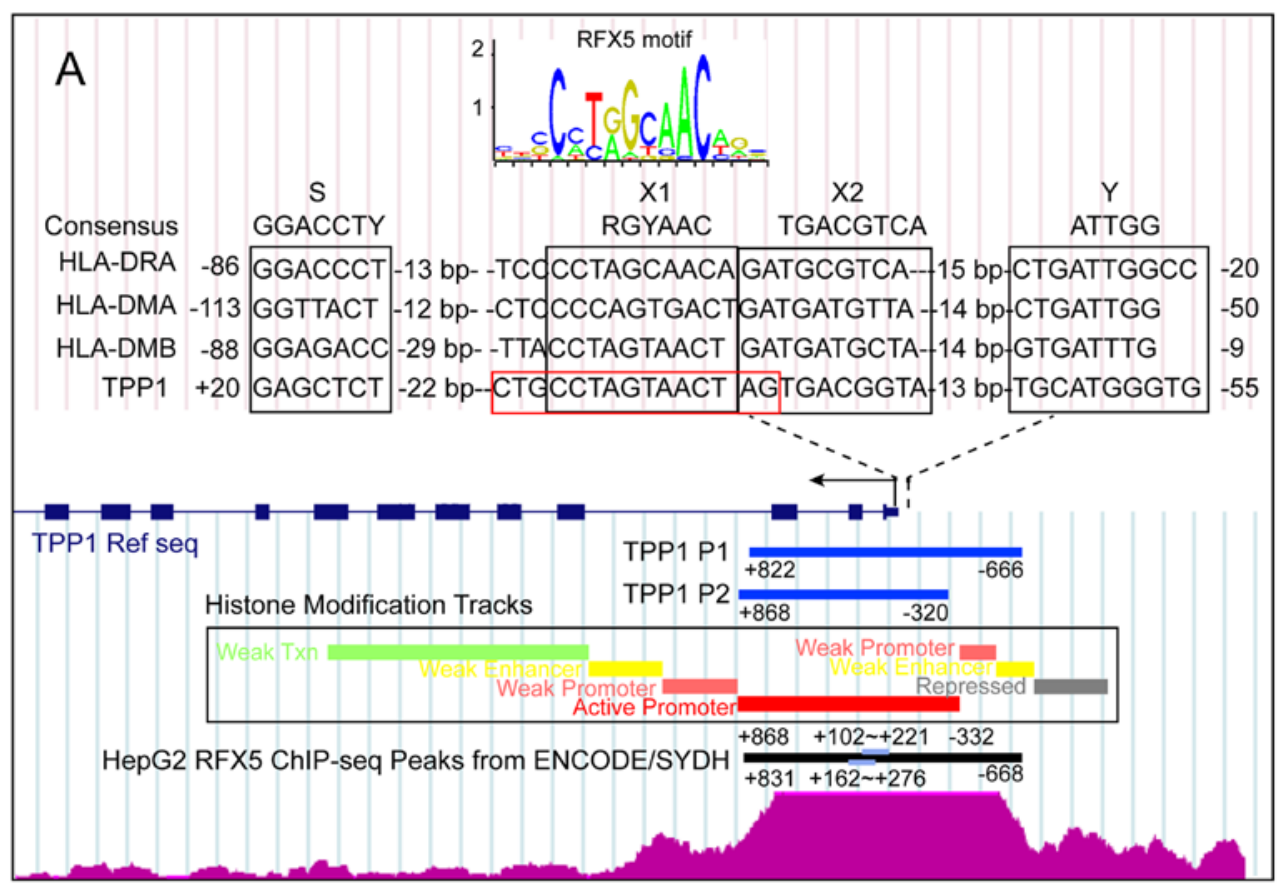

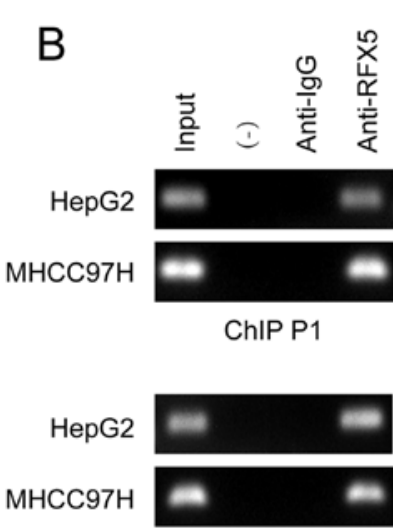

ChIP P2

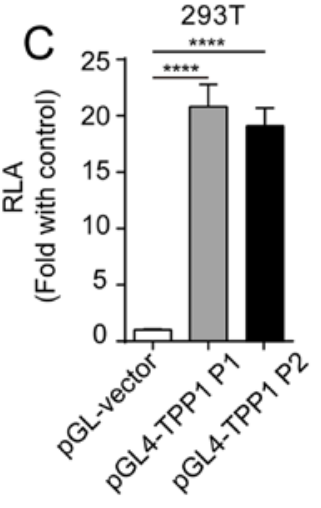

$\mathrm{E}$

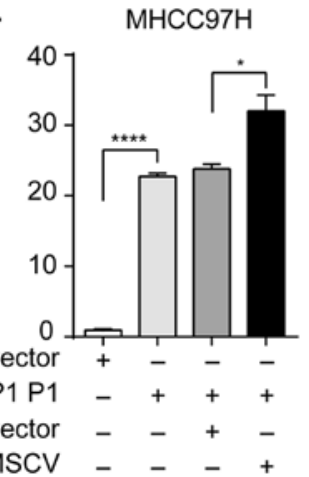

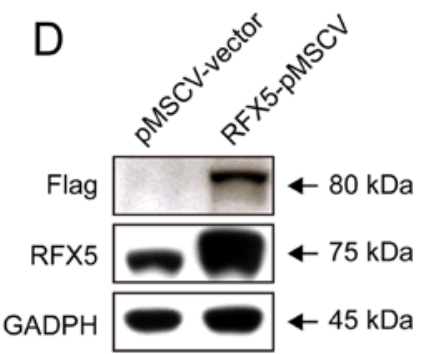

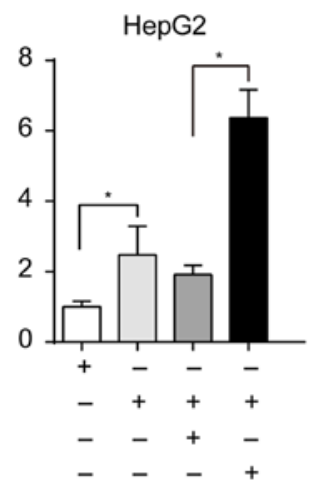

Figure 2. RFX5 binds to the TPP1 promoter region and activates transcription of the TPP1 promoter. (A) The sketch of the TPP1 promoter region shows the RFX5 ChIP-seq binding peak in HepG2 cell and designed primers. Upper, RFX5 motif consensus sequence from ENCODE is shown, the S-Y motifs from HLA-DRA, HLA-DMA, HLA-DMB and TPP1 are boxed. Lower, (blue bold lines) the design of two PCR amplicons for the luciferase reporter gene, (Box) annotation of the TPP1 promoter based on the ENCODE Histone Modification Tracks, (light blue bold lines) the two ChIP-PCR amplicons and (purple peak) the RFX5 ChIP-seq binding peak in the TPP1 promoter. (B) ChIP-PCR assays showing RFX5 binding to the TPP1 promoter in HCC cells. Immunoprecipitated DNA fragments were analyzed by PCR using two independent primers mapped to the RFX5 ChIP-seq binding peak. (C) The transcriptional activity of two TPP1 promoter reporter constructs. Relative luciferase activity (RLA) was calculated as the ratio of firefly to Renilla luciferase activities to represent the promoter activity and performed in triplicate. The results represent one of three experiments. (D) Western blotting showing RFX5 protein with or without overexpression in $293 \mathrm{~T}$ cells. (E) Luciferase assay to analyze the transcriptional impact of RFX5 on the TPP1 promoter in HCC cells. ${ }^{*} \mathrm{P}<0.05$, ${ }^{* * * *} \mathrm{P}<0.0001$.

are highly-conserved in the promoter region of all of the MHCII genes $(8,18,19)$. Most importantly, the TPP1 promoter also contains an $\mathrm{X} 1$ box that is highly consistent with the RFX5 DNA binding motif consensus sequences predicted by the ENCODE ChIP-seq project (Fig. 2A). These results demonstrate that RFX5 protein can directly bind to the TPP1 promoter in $\mathrm{HCC}$ cells and most likely bind to the $\mathrm{X} 1$ box in the TPP1 promoter region. 

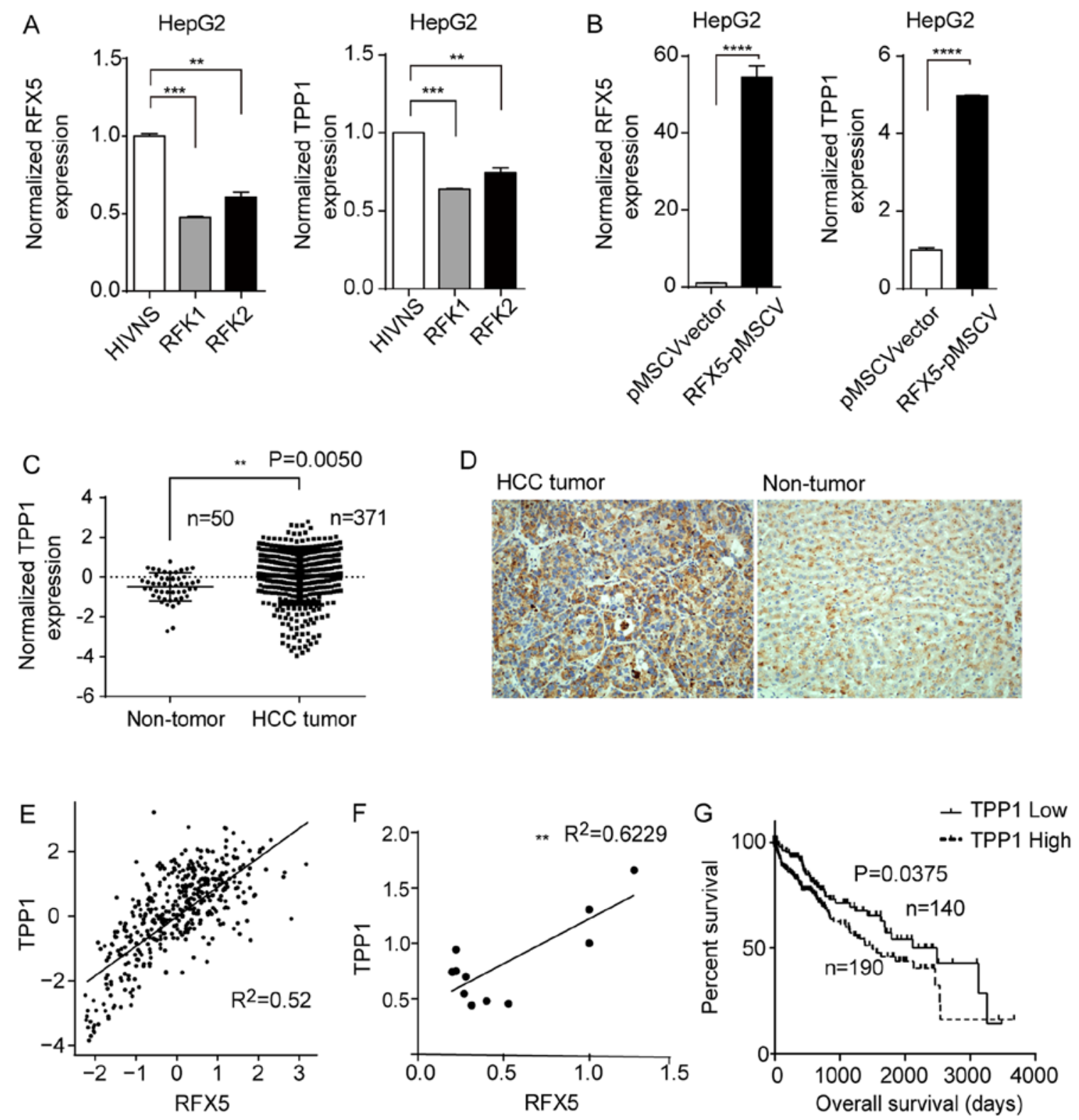

Figure 3. TPP1 expression is linked to RFX5 expression and poor prognoses in HCC. (A) Real-time PCR showing RFX5 and TPP1 expression in HepG2 cells after RFX5 knockdown. (B) Real-time PCR shows RFX5 and TPP1 expression in HepG2 cells after RFX5 overexpression. (C) TPP1 mRNA expression in the TCGA LIHC data determined by RNAseq. (D) Immunohistochemistry staining of TPP1 in HCC and matched adjacent liver tissues by IHC (magnification, x200). (E) Correlation analysis between RFX5 and TPP1 expression from the TCGA LIHC data. (F) Correlation analysis between RFX5 and TPP1 expression using real-time PCR data from the Guilin cohort. (G) Survival analysis from the overall survival time (OST) in HCC patients with relatively lower or higher TPP1 expression in the tumor tissues. The Kaplan-Meier plot was analyzed by the log-rank test. ${ }^{* *} \mathrm{P}<0.01,{ }^{* * *} \mathrm{P}<0.001,{ }^{* * * * *} \mathrm{P}<0.0001$.

RFX5 promotes the transcriptional activity of TPP1 promoter. We further determined whether the binding of RFX5 to the TPP1 promoter could functionally initiate TPP1 gene transcription. Two TPP1 reporters could successfully upregulate the downstream luciferase expression to 20 -fold compared with the control group in 293T cells. The pGL4-TPP1 P1 reporter showed slightly higher transcription levels, so it was used for the following luciferase assays (Fig. 2C). To reconfirm the regulatory effect of RFX5, a Flag-tagged RFX5 expression plasmid was constructed and validated by western blotting with both anti-Flag and anti-RFX5 antibodies (Fig. 2D). Concurrent expression of RFX5 with the TPP1 reporter induced higher luciferase activity in both MHCC97H and HepG2 cells. The increased activation of luciferase was higher in $\mathrm{MHCC} 97 \mathrm{H}$ cells than that in HepG2 cells, which might attribute to higher transfection efficiency (Fig. 2E). These data suggest that RFX5 increases the transcriptional activity of the TPP1 promoter.

TPP1 expression is linked to RFX5 expression and poor prognosis in HCC. We next determined the relationship between RFX5 and TPP1 in HCC cells and tissues. Two independent shRNAs that specifically knock down RFX5 in parallel lowered TPP1 mRNA levels compared with the control group in HepG2 cells (Fig. 3A). Based on the RNAseq TCGA LIHC data from 421 tissues, TPP1 was significantly upregulated in HCC tumors compared with the adjacent non-tumor tissues (Fig. 3C). Immunohistochemistry staining demonstrated that 11 of 14 cases showed TPP1 overexpression in HCC compared 
with matched non-tumor tissues (Fig. 3D). TPP1 mRNA expression increased when RFX5 was transiently overexpressed in HepG2 cells (Fig. 3B). Moreover, RFX5 mRNA expression levels were tightly correlated with that of TPP1 in a large HCC cohort from TCGA with a $\mathrm{R}^{2}$ of 0.52 (Fig. 3E). Similar positive correlation was also observed in HCC samples from Guilin (Fig. 3F). These data suggested that RFX5 could positively modulate TPP1 expression in HCC.

To assess the potential role of TPP1 in HCC patients, we carried out survival analyses in $330 \mathrm{HCC}$ patients from TCGA with survival data. Patients were classified into two groups based on their TPP1 expression levels in tumor tissues. Those patients with a TPP1 expression level higher than mean value were put into the 'TPP1 high' group, while the other patients were put into the 'TPP1 low' group. Multivariate survival analysis of the data in the TCGA cohort suggested that the expression level of TPP1 is not an independent prognostic indicator for survival of HCC patients (data not shown). However, Kaplan-Meier survival analysis showed that patients with relatively lower TPP1 mRNA expression had longer overall survival times than patients with higher TPP1 mRNA expression levels (Fig. 3G).

\section{Discussion}

Our study identified that RFX5 is overexpressed in HCC and acts as a transcriptional activator of the non-MHCII gene TPP1 in HCC. In previous studies, as a transcriptional factor encoding a DNA binding protein, RFX5 has been revealed to be a crucial MHCII gene transcription regulator. RFX5 mutations that result in severely truncated or absent proteins could lead to MHCII transcriptional deficiency (also called the bare lymphocyte syndrome, BLS), a severe immunodeficiency syndrome $(3,20)$. However, our present study identified that overexpressed RFX5 in HCC tumor tissues and cell lines does not elevate MHCII expression. The heterogeneous and sparse expression of MHCII genes in HCC tumors, adjacent tissues and cell lines were also observed in previous studies and mainly attributed to mesenchymal cells in the liver sinusoid, not HCC cells (21-23). Hence, the overexpressed RFX5 in HCC may play a role in the tumor by transactivating nonMHCII target genes, such as TPP1.

Our data showed that RFX5 could directly bind to the TPP1 promoter and most likely binds to the $\mathrm{X} 1$ box in the TPP1 promoter region by ChIP-seq and ChIP-PCR assay. We also proved that transient overexpression of RFX5 could increase the transcriptional levels of functionally activated TPP1 promoter by luciferase assay and modulate the expression of RFX5 in HCC cell lines, subsequently altering TPP1 expression levels. Strikingly, TPP1 mRNA and protein expression levels were elevated in HCC, and the mRNA levels were tightly correlated with that of RFX5 in two independent HCC cohorts. Most importantly, patients with higher TPP1 expression levels in HCC tissues tend to have shorter overall survival times. Although TPP1 expression may not be a good prognostic maker for HCC patients, at least in the TCGA cohort, its upregulation transactivated by RFX5 overexpression may contribute to the progress of HCC.

The above finding further supports previous studies that also identified TPP1 as a potential transcriptional target of
CIITA, given that CIITA and RFX5 closely collaborate in transactivating MHCII genes $(18,24)$. Moreover, RFX5 is located in 1q21, a region frequently involved in chromosomal aberrations in preneoplastic and neoplastic diseases (25). Together, these data indicate that RFX5 itself or its downstream target genes might have more global roles in neoplastic diseases. However, the detailed molecular mechanism for RFX5 in TPP1 transcriptional regulation and its role in HCC carcinogenesis needs to be explored further.

In conclusion, this study showed that RFX5 is upregulated in HCC and transactivates the non-MHCII gene TPP1, which can lead to TPP1 overexpression and could have biological implications in HCC.

\section{Acknowledgements}

This study was supported by grants from the National Key Sci-Tech Special Project of China (2012ZX10002011-006), Chinese National Natural Science Foundation Grant (81541151, $81201569,81171550,81101302,81273202)$, the Beijing Natural Science Foundation (7132186), Clinical Medicine Science and Technology Project of Jiangsu Province of China (BL2013024) and Jiangsu Provincial Key Research and Development Program (BE2016721).

\section{References}

1. Emery P, Durand B, Mach B and Reith W: RFX proteins, a novel family of DNA binding proteins conserved in the eukaryotic kingdom. Nucleic Acids Res 24: 803-807, 1996.

2. Aftab S, Semenec L, Chu JS and Chen N: Identification and characterization of novel human tissue-specific RFX transcription factors. BMC Evol Biol 8: 226, 2008.

3. Steimle V, Durand B, Barras E, Zufferey M, Hadam MR, Mach B and Reith W: A novel DNA-binding regulatory factor is mutated in primary MHC class II deficiency (bare lymphocyte syndrome). Genes Dev 9: 1021-1032, 1995.

4. Durand B, Kobr M, Reith W and Mach B: Functional complementation of major histocompatibility complex class II regulatory mutants by the purified X-box-binding protein RFX. Mol Cell Biol 14: 6839-6847, 1994.

5. Garvie CW and Boss JM: Assembly of the RFX complex on the MHCII promoter: Role of RFXAP and RFXB in relieving autoinhibition of RFX5. Biochim Biophys Acta 1779: 797-804, 2008.

6. Moreno CS, Beresford GW, Louis-Plence P, Morris AC and Boss JM: CREB regulates MHC class II expression in a CIITAdependent manner. Immunity 10: 143-151, 1999.

7. Hanna S and Etzioni A: MHC class I and II deficiencies. J Allergy Clin Immunol 134: 269-275, 2014.

8. Ting JP and Trowsdale J: Genetic control of MHC class II expression. Cell 109 (Suppl): S21-S33, 2002.

9. Gobin SJ, Peijnenburg A, van Eggermond M, van Zutphen M, van den Berg R and van den Elsen PJ: The RFX complex is crucial for the constitutive and CIITA-mediated transactivation of MHC class I and beta2-microglobulin genes. Immunity 9: 531-541, 1998.

10. Sengupta PK, Fargo J and Smith BD: The RFX family interacts at the collagen (COL1A2) start site and represses transcription. J Biol Chem 277: 24926-24937, 2002.

11. Di Giacopo R, Cianetti L, Caputo V, La Torraca I, Piemonte F, Ciolfi A, Petrucci S, Carta C, Mariotti P, Leuzzi V, et al: Protracted late infantile ceroid lipofuscinosis due to TPP1 mutations: Clinical, molecular and biochemical characterization in three sibs. J Neurol Sci 356: 65-71, 2015.

12. Sleat DE, Donnelly RJ, Lackland H, Liu CG, Sohar I,Pullarkat RK and Lobel P: Association of mutations in a lysosomal protein with classical late-infantile neuronal ceroid lipofuscinosis. Science 277: 1802-1805, 1997.

13. ENCODE Project Consortium: A user's guide to the encyclopedia of DNA elements (ENCODE). PLoS Biol, 2011. http://dx.doi. org/10.1371/journal.pbio.1001046. Accessed April 19, 2011. 
14. Mardis ER: ChIP-seq: Welcome to the new frontier. Nat Methods 4: 613-614, 2007.

15. Dunham I, Kundaje A, Aldred SF, Collins PJ, Davis CA, Doyle F, Epstein CB, Frietze S, Harrow J, Kaul R, et al; ENCODE Project Consortium: An integrated encyclopedia of DNA elements in the human genome. Nature 489: 57-74, 2012.

16. Maher B: ENCODE: The human encyclopaedia. Nature 489: 46-48, 2012.

17. ENCODE Project Consortium: The ENCODE (ENCyclopedia of DNA elements) project. Science 306: 636-640, 2004.

18. Krawczyk M, Seguín-Estévez Q, Leimgruber E, Sperisen P, Schmid C, Bucher P and Reith W: Identification of CIITA regulated genetic module dedicated for antigen presentation. PLoS Genet 4: e1000058, 2008.

19. Westerheide SD, Louis-Plence P, Ping D, He XF and Boss JM: HLA-DMA and HLA-DMB gene expression functions through the conserved S-X-Y region. J Immunol 158: 4812-4821, 1997.

20. Nekrep N, Fontes JD, Geyer M and Peterlin BM: When the lymphocyte loses its clothes. Immunity 18: 453-457, 2003.

21. Paterson AC, Sciot R, Kew MC, Callea F, Dusheiko GM and Desmet VJ: HLA expression in human hepatocellular carcinoma. Br J Cancer 57: 369-373, 1988.
22. Xie XW, Mei MH, Liao WJ, Qian LH, Yu X, Fei R, Qin LL, Zhang HH, Peng JR, Shen DH, et al: Expression of CIITA-related MHCII molecules in tumors linked to prognosis in hepatocellular carcinoma. Int J Oncol 34: 681-688, 2009.

23. Sung CH, Hu CP, Hsu HC, Ng AK, Chou CK, Ting LP, Su TS, Han SH and Chang CM: Expression of class I and class II major histocompatibility antigens on human hepatocellular carcinoma. J Clin Invest 83: 421-429, 1989.

24. Wong D, Lee W, Humburg P, Makino S, Lau E, Naranbhai V, Fairfax BP, Chan K, Plant K and Knight JC: Genomic mapping of the MHC transactivator CIITA using an integrated ChIP-seq and genetical genomics approach. Genome Biol 15: 494, 2014.

25. Villard J, Reith W, Barras E, Gos A, Morris MA, Antonarakis SE, Van den Elsen PJ and Mach B: Analysis of mutations and chromosomal localisation of the gene encoding RFX5, a novel transcription factor affected in major histocompatibility complex class II deficiency. Hum Mutat 10: 430-435, 1997. 